\title{
Patología oculoplástica: Experiencia de 7 años en Hospital del Sistema de Salud Público de Chile
}

\author{
Michel Olivera I., ${ }^{1,3}$ José Luis Román F.,3, Rubén Suárez A., \\ Pablo Troncoso ${ }^{1,3}$ y Gely Muñoz B. ${ }^{2,3}$
}

\section{Oculoplastic Pathology: Experience of 7 years in Hospital of the Public Health System of Chile}

Introduction: The oculoplastic surgery studies and treats disorders of the eyelids, orbit and lacrimal ways. Among the most important pathologies by frequency and impact are: palpebral ptosis, blepharochalasis, ectropion and entropion. Aim: Analyze the experience of El Pino Hospital regarding the management of oculoplastic pathology in the hands of a plastic surgeon.Materials and Method: Descriptive and retrospective study. We reviewed files of patients operated with diagnosis of blepharochalasis, palpebral ptosis, ectropion and entropion between the years 2010 and 2017. Data were analyzed as age, sex, comorbidities, cause of the defect, oculoplastic surgery, type of anesthesia, postoperative complication, follow-up time and results. Results: Between the years 2010 and 2017, 79 patients with oculoplastic pathology operated, 17 for palpebral ptosis, 42 for blefarochalasis, 14 for ectropion and 6 for entropion, being the main senile cause $(93,7 \%)$. The surgeries performed were: elevator pexis for palpebral ptosis, blepharoplasty for blefarochalasis and for ectropion-entropion tarsal strip. The main anesthetic technique used was local anesthesia + sedation $(54,4 \%)$. Among the total postoperative complications, we have: lagophthalmos $(2$ cases), residual ectropion ( 3 cases), conjunctivitis (3 cases), wound dehiscence ( 2 cases) and palpebral hematoma (1 case). Six patients were reoperated due to complications. The results obtained according to the subjective evaluation of the plastic surgeon were regular (5 cases), good (20 cases) and very good (54 cases). Conclusion: Management of oculoplastic pathology requires mostly ambulatory surgeries with good results and low morbidity, which is feasible to perform in a public hospital. We believe it is necessary to use some objective instrument to better evaluate the palpebral defects and their correction.

Key words: oculoplastic pathology; blepharochalasis; palpebral ptosis; blepharoplasty; pexia levator.

\section{Resumen}

Introducción: La cirugía oculoplástica estudia y trata los trastornos de párpados, órbita y vías lagrimales. Entre las patologías más importantes por frecuencia e impacto tenemos: ptosis palpebral, blefarochalasis, ectropión y entropión. Objetivo: Analizar la experiencia del Hospital El Pino en cuanto al manejo de patología oculoplástica en manos de un cirujano plástico. Materiales y Método: Estudio descriptivo y retrospectivo. Se revisaron fichas de pacientes operados con diagnóstico de blefarochalasis, ptosis palpebral, ectropión y entropión entre los años 2010 y 2017. Se analizaron datos como edad, sexo, comorbilidades, causa del defecto, cirugía oculoplástica, tipo de anestesia, complicación posoperatoria, tiempo de seguimiento y resultados. Resultados: Entre los años 2010 y 2017 se operaron 79 pacientes con patología oculoplástica, 17 por ptosis palpebral, 42 por blefarochalasis, 14 por ectropión y 6 por entropión, siendo la principal causa senil $(93,7 \%)$. Las cirugías realizadas fueron: pexia del elevador para Ptosis palpebral, blefaroplastía para blefarochalasis y para ectropión-entropión tarsal strip. La principal técnica anestésica utilizada fue anestesia local + sedación $(54,4 \%)$. Entre el total de complicaciones posoperatorias tenemos: lagoftalmo ( 2 casos), ectropión residual ( 3 casos), conjuntivitis ( 3 casos), dehiscencia de herida ( 2 casos) y hematoma palpebral (1 caso). Se reintervinieron 6 pacientes por complicaciones. Los resultados obtenidos según la evaluación subjetiva del cirujano plástico y pacientes fueron regular ( 5 casos), bueno (20 casos) y muy bueno (54 casos). Conclusión: El manejo de la patología oculoplástica requiere en su mayoría de cirugías ambulatorias con buenos resultados y poca morbilidad, lo cual es factible de realizar en un hospital público. Creemos necesario el uso de algún instrumento objetivo para evaluar de mejor manera los defectos palpebrales y su corrección.

Palabras clave: patología oculoplástica; blefarochalasis; ptosis palpebral; blefaroplastía; pexia elevador.

\author{
'Servicio de Cirugía Hospital El \\ Pino. Santiago, Chile. \\ 2Interna de Medicina, \\ ${ }^{3}$ Facultad de Medicina \\ Universidad Andrés Bello.
}

Recibido el 15 de enero de 2018 y aceptado para publicación el 6 de julio de 2018.

Correspondencia a: Dr. Michel Olivera I. mjrules23@hotmail.com 


\section{Introducción}

La cirugía oculoplástica es una subespecialidad de la cirugía plástica que estudia y trata los trastornos de párpados, órbita y vías lagrimales. En todos los tratamientos busca la mejora estética del paciente y, lo que es más importante, la recuperación de la función del ojo y sus estructuras anexas ${ }^{1,2}$.

Entre las patologías oculoplásticas más importantes por su frecuencia e impacto se encuentran: Ptosis palpebral (caída de los párpados), blefarochalasis (exceso de piel en los párpados), ectropión (eversión del borde del párpado) y entropión (inversión del borde del párpado) cuyas causas pueden ser congénitas o adquiridas (senil o involutiva, mecánica $o$ traumática, cicatricial, neurogénica) ${ }^{2}$.

El objetivo de nuestro estudio es dar a conocer la frecuencia diagnóstica y las soluciones propuestas para el manejo de pacientes con patología oculoplástica que consultan en nuestro hospital.

\section{Materiales y Métodos}

Se diseñó un estudio descriptivo, retrospectivo y observacional mediante la revisión de fichas clínicas que incluyeran pacientes con diagnóstico de blefarochalasis, ptosis palpebral, ectropión y entropión evaluados en policlínico de cirugía plástica del Hospital El Pino entre los años 2010 y 2017. No se utilizaron criterios de exclusión.

Entre los datos analizados se incluyen: edad, sexo, comorbilidades, tabaco, causa del defecto (congénito o adquirido), tratamiento previo, evaluación con campimetría, tipo de cirugía oculoplástica, tipo de anestesia, complicación posoperatoria y su tratamiento, tiempo de primer control, tiempo de seguimiento y resultados.

Todas las evaluaciones de pacientes y procedimientos quirúrgicos fueron realizadas por un único cirujano plástico. La evaluación clínica para blefarochalasis y blefaroptosis se realiza considerando la distancia de reflejo marginal (MRD), pliegue superior del párpado y campimetría de Goldmann ${ }^{1}$ en casos severos y bilaterales. En los casos de ectropión y entropión utilizamos snap back test $\mathrm{y}$ test de distracción ${ }^{2}$.

La evaluación de los resultados se realizó de manera subjetiva de acuerdo a la satisfacción del paciente y del propio cirujano plástico.

\section{Resultados}

Entre los años 2010 y 2017 se operaron 79 pacientes por patología oculoplástica con edades entre 18 y 87 años (63,9 años). El $62 \%$ de los pacientes eran mujeres, las principales comorbilidades fueron hipertensión arterial, diabetes mellitus 2, artritis reumatoide, hipotiroidismo y posoperatorio de catarata; solo el $36 \%$ de los pacientes presentaba hábito tabáquico (Tabla 1).

Las patologías oculoplásticas operadas fueron: Ptosis palpebral (17 casos), blefarochalasis (42 casos), ectropión (14 casos) y entropión (6 casos) siendo las causas de ellas senil o involutiva $(93,7 \%)$, posquirúrgica o traumática $(5 \%)$ y congénita $(1,3 \%)$ en un caso de ptosis palpebral (Tabla 2).

Sólo dos pacientes recibieron tratamiento previo por patología oculoplástica: botox en un caso de blefaroptosis bilateral y sutura más plastía palpebral por herida traumática palpebral superior con lagof-

Tabla 1. Características de pacientes con patología oculoplástica

\begin{tabular}{|lll|}
\hline Sexo & Femenino: 49 & \\
& Masculino: 30 & \\
Rango etario & 18 a 87 años (edad promedio 63,9 años) & $43 \%$ \\
Comorbilidades & Hipertensión arterial & $11,1 \%$ \\
& Diabetes mellitus 2 & $6,9 \%$ \\
& Hipotiroidismo & $5,6 \%$ \\
& Catarata & $4,2 \%$ \\
& Accidente cerebrovascular & $4,2 \%$ \\
Tabaquismo & Enfermedad pulmonar obstructiva crónica & \\
& Sí: 29 & \\
Total de pacientes & No: 50 \\
\hline
\end{tabular}


Tabla 2. Patologías oculoplásticas y causas

\begin{tabular}{|lll|}
\hline $\begin{array}{l}\text { Tipo de patología } \\
\text { oculoplástica }\end{array}$ & Causa & Total \\
\hline $\begin{array}{l}\text { Blefarochalasis } \\
\text { Ptosis Palpebral }\end{array}$ & $\begin{array}{l}\text { Senil: } 42 \text { casos } \\
\text { Senil: } 15 \text { casos } \\
\text { Posquirúrgica: } 1\end{array}$ & 42 casos \\
Congénita: 1 & 17 casos \\
\hline Ectropión & $\begin{array}{l}\text { Senil: } 12 \text { casos } \\
\text { Posquirúrgico: } 1 \text { caso } \\
\text { Traumática: } 1 \text { caso }\end{array}$ & \\
\hline Entropión & Senil: 6 casos & 6 casos \\
\hline
\end{tabular}

talmo. La evaluación con campimetría se utilizó en casos severos y bilaterales de blefarochalasis ( 7 casos), ptosis palpebral (3 casos) y ectropión (1 caso).

Las cirugías realizadas por patología fueron: blefaroplastía (superior uni y bilateral, inferior bilateral y de cuatro párpados) para blefarochalasis (Figura 1), pexia del elevador para ptosis palpebral (Figura 2) y para ectropión-entropión tarsal strip, tarsal strip asociado a colgajo de Tripier (1 caso) y resección de retractores (3 casos) (Figuras 3 y 4). La técnica anestésica utilizada para la mayoría de los procedimientos fue anestesia local + sedación $(54,4 \%)$ (Tabla 3).

Hubo 22 complicaciones posoperatorias $(27,8 \%)$, siendo las principales: sensación de cuerpo extraño ( 2 casos) para pexia del elevador; lagoftalmo (2 casos), equimosis (1 caso), edema palpebral (2 casos), exceso cutáneo (1 caso), ptosis (1 caso) para blefaroplastía y ectropión residual (3 casos), conjuntivitis (4 casos), dehiscencia de sutura (2 casos), hematoma palpebral (1 caso) y cicatriz retráctil (1 caso) en caso de tarsal strip. Se reintervinieron 8 pacientes por complicaciones realizándose retiro de puntos por lagoftalmo (2 casos), corrección tarsal strip inferior (1 caso) y tarsal strip + colgajo de tripier de novo por ectropión residual (1 caso) (Figura $5)$, hemostasia por hematoma palpebral (1 caso), retiro de retractores inferiores por entropión (1 caso), electrofulguración de pestañas por triquiasis (1 caso) y corrección de tarsal strip por ectropión (1 caso) (Tabla 4).

El primer control posoperatorio se realizó entre 6 y 60 días (debido principalmente a inasistencia de pacientes a control y reagendamiento de citación), tiempo de seguimiento de 14 días a 1 año 1 mes. Los resultados obtenidos de acuerdo a la evaluación del cirujano plástico y satisfacción de pacientes fueron regular en 5 casos, bueno 20 casos y muy bueno 54 casos.

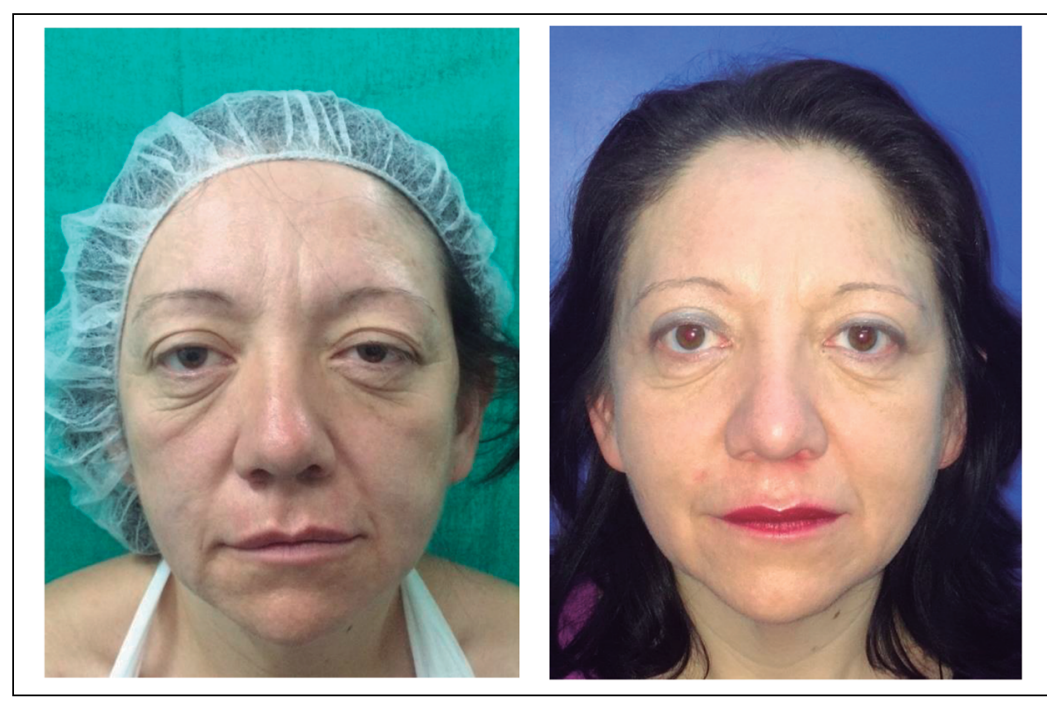

Figura 1. Blefarochalasis palpebral superior bilateral. Pre y posoperatorio.

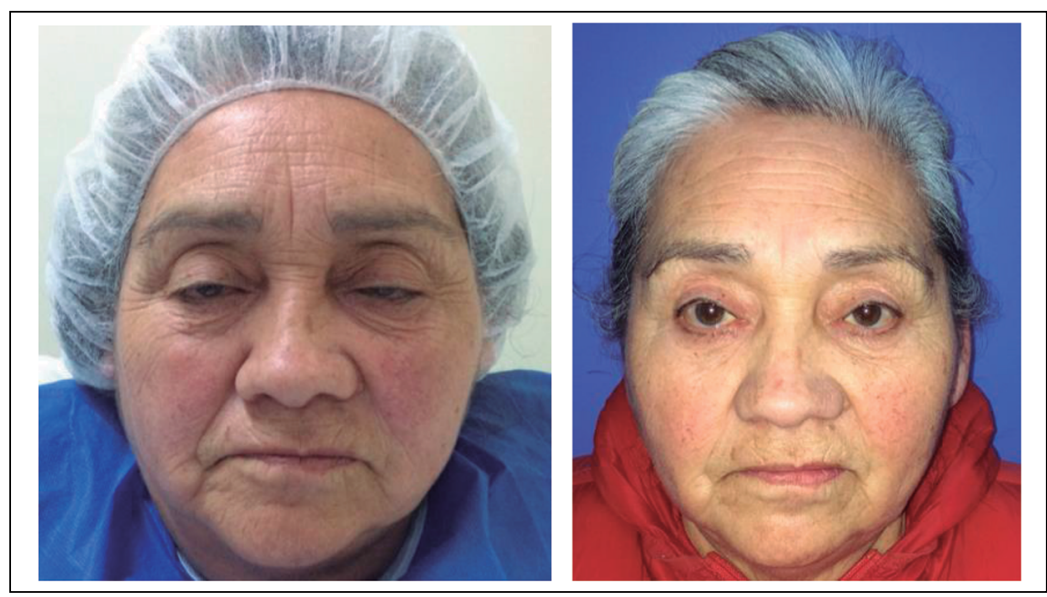

Figura 2. Ptosis palpebral superior bilateral. Pre y posoperatorio.

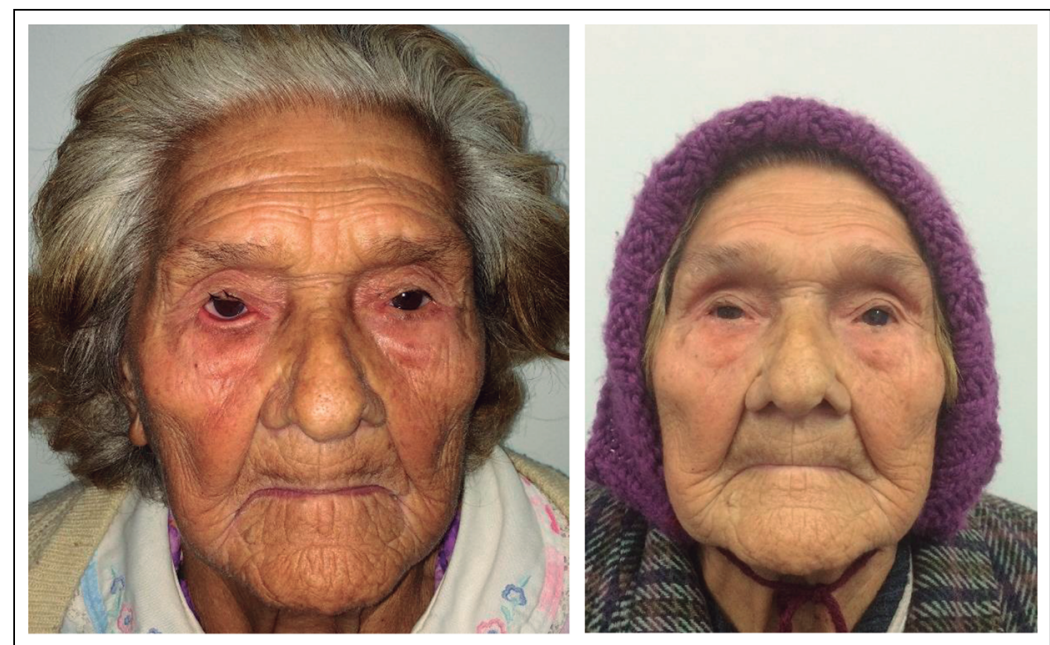

Figura 3. Ectropión. Pre y posoperatorio. 


\section{ARTíCULO ORIGINAL}

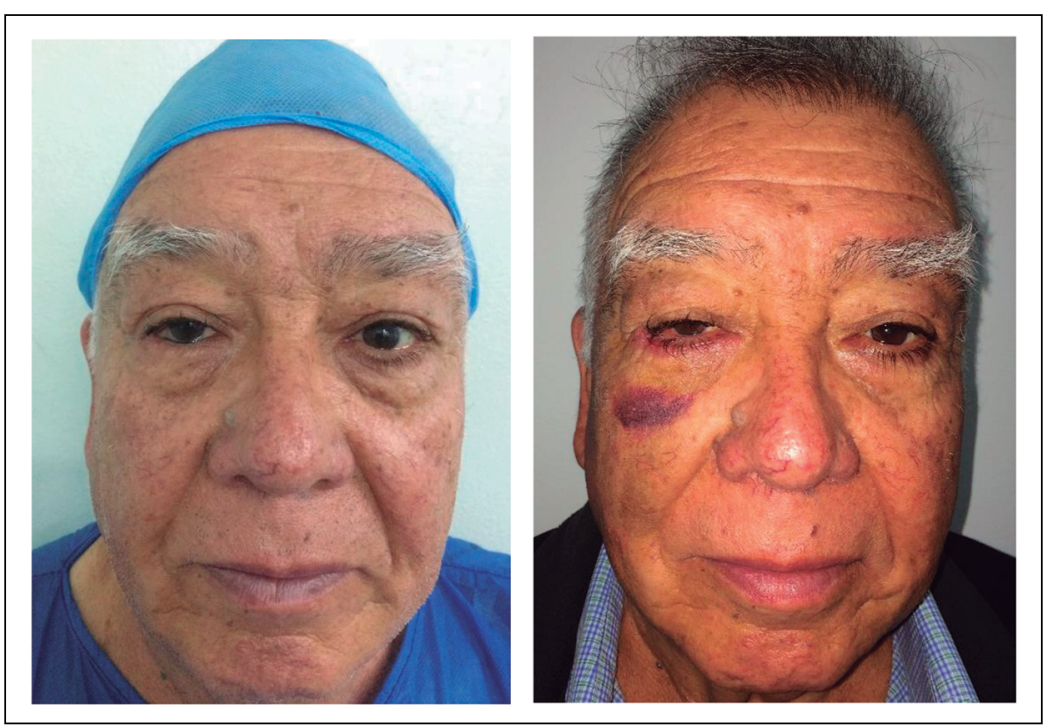

Figura 4. Entropión. Pre y posoperatorio.

\section{Discusión}

La mayoría de los artículos revisados en este trabajo dan cuenta de la mayor frecuencia de blefarochalasis y blefaroptosis como patologías oculoplásticas predominantes, lo cual se correlaciona con nuestra experiencia (42 casos blefarochalasis, 17 casos blefaroptosis $)^{2}$.

Respecto a la etiología de las patologías analizadas, la literatura las clasifica en congénitas y adquiridas (senil o involutiva, neurogénica, miogénica, traumática, posquirúrgica $)^{2,3}$ siendo estas últimas las de mayor relevancia. Dentro de la etiología adquirida destaca como principal causa la involutiva o senil que, en el caso de la blefaroptosis, se debe a un estiramiento gradual o dehiscencia de la aponeurosis del músculo elevador del párpado ${ }^{4}$, y en el caso del ectropión-entropión involucran la laxitud palpebral horizontal por debilitamiento cantal, insuficiencia de

Tabla 3. Tratamiento de patología oculoplástica y resultados

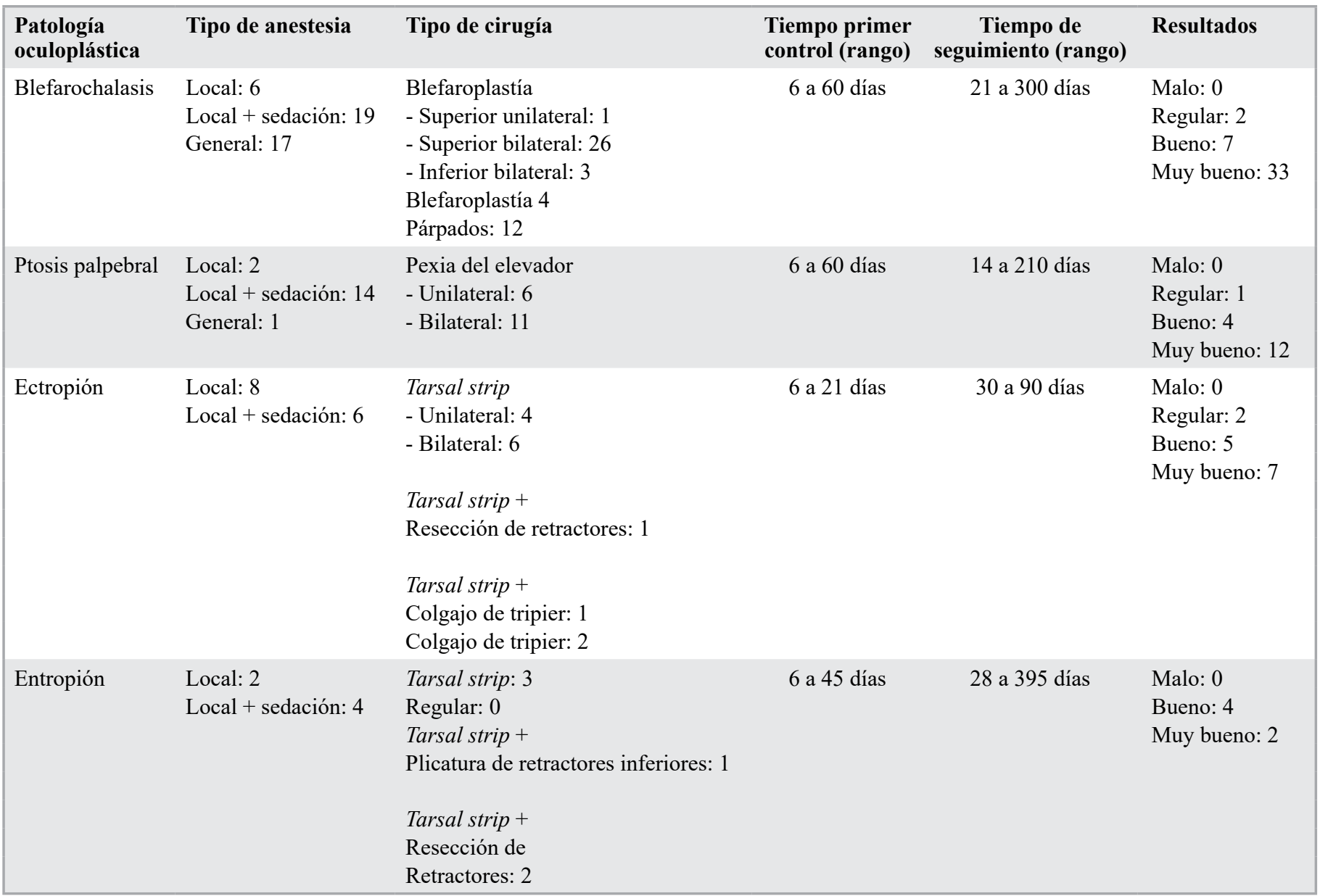




\section{ARTÍCULO ORIGINAL}

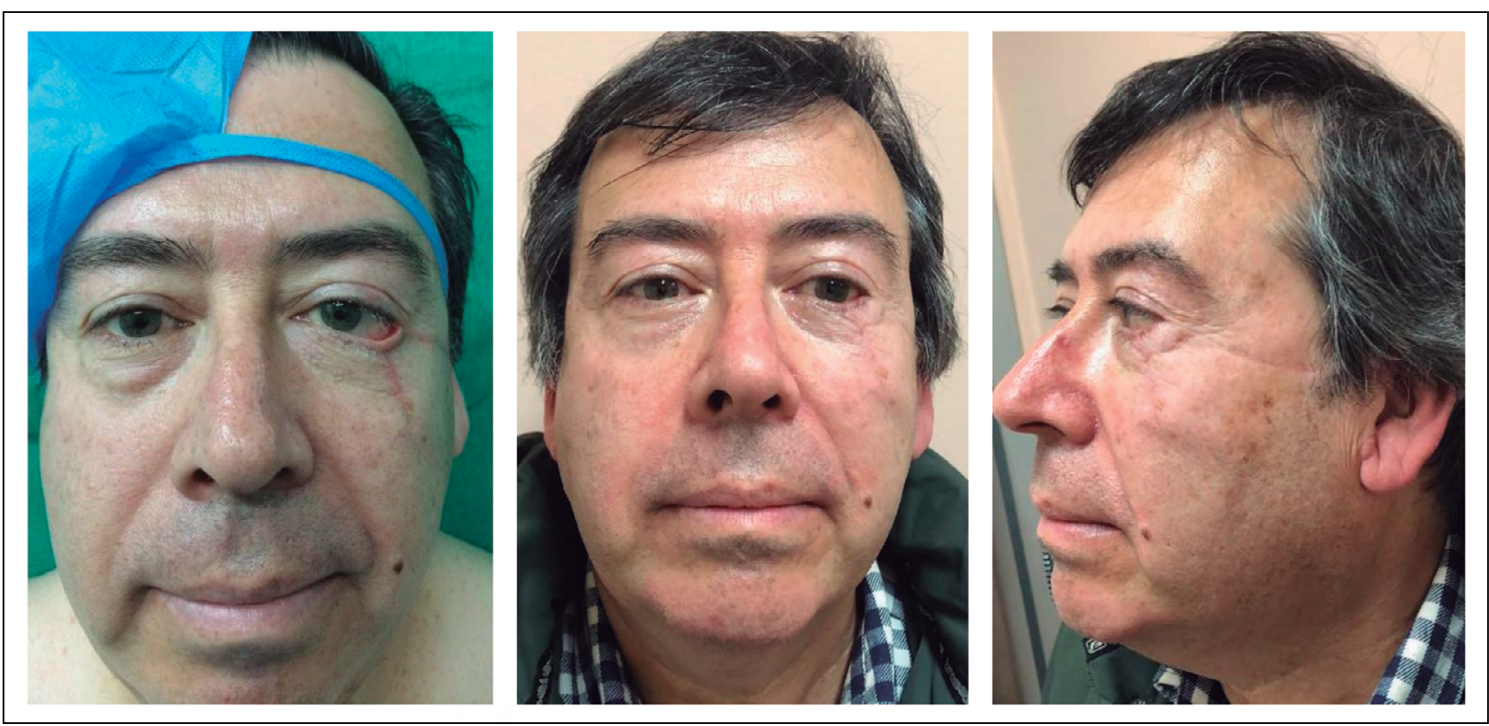

Figura 5. Complicación posoperatoria de reparación ectropión + resección carcinoma basocelular periocular izquierdo. El manejo incluyó tarsal strip + colgajo de Tripier de novo.

Tabla 4. Complicaciones posoperatorias y tratamiento

\begin{tabular}{|lll|}
\hline Complicación posoperatoria & Tratamiento de complicación & Número de casos \\
Conjuntivitis & $\begin{array}{l}\text { Colirio de cloranfenicol } \\
\text { Colirio de tobramicina + dexametasona }\end{array}$ & 4 \\
\hline Ectropión & Corrección tarsal strip inferior, nuevo tarsal strip + colgajo de tripier & 3 \\
\hline Lagoftalmo & Retiro de puntos & 2 \\
\hline Sensación de cuerpo extraño & Retiro de puntos & 2 \\
\hline Dehiscencia de sutura & Colirio de cloranfenicol, afrontamiento con Steri-Strip ${ }^{\circledR}$ & 2 \\
Edema palpebral & Masaje & 2 \\
\hline Hematoma palpebral & Revisión de hemostasia & 1 \\
Cicatriz retráctil & Infiltración con metilprednisolona & 1 \\
\hline Equimosis & Masaje con arnica & 1 \\
\hline Entropión persistente & Retiro retractores inferiores & 1 \\
\hline Triquiasis & Electrofulguración de pestañas & 1 \\
\hline Ptosis palpebral leve & & 1 \\
\hline Exceso cutáneo & & \\
\hline
\end{tabular}

los retractores del párpado inferior, hernia de grasa orbitaria, alteraciones del músculo orbicular de los párpados $^{5}$. En nuestra serie las principales causas, analizadas globalmente, dan cuenta de un 93,7\% para etiología senil o involutiva, 5\% posquirúrgica o traumática y $1,3 \%$ congénita.

Para el diagnóstico de los pacientes con blefarochalasis y blefaroptosis se deben evaluar al menos 4 mediciones $^{4}$ : Altura de la fisura palpebral, distancia de reflejo marginal (MRD), Pliegue superior del párpado y Prueba de la Función del Elevador (LF).

Otra forma en que la ptosis palpebral se cuantifica comúnmente es trazar el área de privación del campo visual que resulta de la mala posición del párpado mediante la realización de la campimetría de Goldmann, una vez con el párpado en su posición natural y de nuevo con el párpado grabado para simular los resultados de la corrección quirúrgica ${ }^{6}$. 
La evaluación clínica de ectropión y entropión implica explorar dos factores: Exploración de la laxitud palpebral inferior (mediante snap back test y test de distracción) y exploración de la insuficiencia de los retractores (que se manifiesta por ausencia del pliegue palpebral inferior, un fondo de saco conjuntival estrecho y muy profundo) y una pérdida de la excursión normal del párpado inferior en la mirada hacia abajo (ptosis del párpado inferior) ${ }^{5}$.

Dicho esto, nuestra evaluación clínica para blefaroptosis se basó en la medición de distancia de reflejo marginal, pliegue superior del párpado y en unos pocos casos la campimetría de Goldman; para los casos de ectropión y entropión utilizamos snap back test y test de distracción.

Con respecto al procedimiento quirúrgico, la técnica anestésica de preferencia fue la combinación de anestesia local más sedación (54,4\% de los casos) utilizando como anestésico lidocaína al $2 \%$ con epinefrina 1:100.000 tal como se describe en la literatura ${ }^{7}$. En cuanto a la técnica quirúrgica en sí, para blefarochalasis utilizamos la técnica de blefaroplastía cuyo objetivo es reducir el colgamiento excesivo del pliegue del párpado por resección cutánea, con o sin reducción de la grasa orbitaria ${ }^{8}$, en el caso de blefaroptosis se realiza pexia del músculo elevador del párpado a través de una incisión similar a la blefaroplastía realizando la sección de una tira del músculo orbicular exponiendo la aponeurosis del músculo elevador con posterior sección de la misma y sutura de su borde al tarso ${ }^{9}$. Para el caso de ectropión y entropión utilizamos la técnica de tarsal strip la cual consiste en disecar una porción de tarso lateral y suturarla a nivel del canto lateral ${ }^{10}$.

Como toda área quirúrgica, la oculoplástica no está exenta de complicaciones; afortunadamente estas son de baja incidencia y algunas raras. Según reportes de la literatura las complicaciones pueden fluctuar entre un 0 a $21 \%$ dependiendo de la patología y procedimiento realizado ${ }^{8}$. Entre las principales complicaciones podemos mencionar hematoma retrobulbar y pérdida visual $(1: 10.000)$, ojo seco, lagoftalmo, epífora, blefaroptosis, quemosis conjuntival, ectropión y entropión recidivante, infección, pérdida neurosensorial periorbitaria ${ }^{11}$. Nuestra serie da cuenta de un $27,8 \%$ de complicaciones en global (17,6\% en pexia del elevador, $16,6 \%$ para blefaroplastía y $50 \%$ en tarsal strip) siendo la mayoría complicaciones menores destacando lagoftalmo, ectropión residual, conjuntivitis y hematoma palpebral siendo necesario una nueva intervención quirúrgica en 7 casos.

Los resultados obtenidos, basados en la satisfacción del paciente y evaluación del cirujano plástico, fueron buenos en un $25,3 \%$ y muy buenos en un $68,3 \%$ lo que da un resultado positivo total de $93,6 \%$ que se correlaciona con lo obtenido en otras series. Un aspecto desfavorable al momento de evaluar los resultados es el hecho de basarse en aspectos subjetivos más que en la medición de ciertos parámetros posoperatorios como distancia de reflejo marginal, altura de la fisura palpebral y test de distracción entre otros. En este sentido para estandarizar la medición de resultados sería útil incorporar herramientas como el Inventario de Beneficios de Glasgow (GBI) 10 el cual es un cuestionario posintervencional validado, desarrollado inicialmente para procedimientos otorrinolaringológicos y que actualmente tiene su aplicación en otras áreas quirúrgicas ${ }^{12}$.

\section{Conclusiones}

La patología oculoplástica se hace cada día más frecuente, siendo las alteraciones del párpado su principal campo de acción. Su manejo requiere de procedimientos quirúrgicos ambulatorios con un alto porcentaje de éxito y poca morbilidad, lo cual es factible de realizar en un hospital público, logrando un impacto en la calidad de vida de nuestros pacientes.

Creemos necesario el uso de algún instrumento objetivo para evaluar de mejor manera los defectos palpebrales y corrección de dichos defectos.

\section{Responsabilidades éticas}

Protección de personas y animales. Los autores declaran que para esta investigación no se han realizado experimentos en seres humanos ni en animales.

Confidencialidad de los datos. Los autores declaran que en este artículo no aparecen datos de pacientes.

Conflictos de interés: no hay. 


\section{Referencias}

1. Corrales Negrin Yusleydi, Pérez González Henry, Agramonte Centelles Ileana. Un acercamiento a la historia de la Cirugía Plástica Ocular en Pinar del Río. Rev Ciencias Médicas [Internet]. 2016 Feb [citado el 14 de junio de 2018]; 20(1): Disponible en: http://scielo.sld.cu/scielo. php?script=sci_arttext\&pid=S156131942016000100022\&lng=es.

2. Fontbona M. Experiencia en Cirugía Oculoplástica. Revista Chilena de Cirugía Plástica 2008;6:47-55.

3. Fontbona M, Cabello R, Calderón W, Calderón D, Guerra C, Israel G. Manejo de la ptosis palpebral. Revista Chilena de Cirugía Plástica 2005;3:81-4.

4. Yadegari S. Approach to a patient with blepharoptosis, Neurol Sci. 2016; 37:1589-96.

5. Masanet M, Cervera M. Cirugía del Ectropión y Entropión Senil. En: Laiseca A, Cervera M, Laiseca D. Capítulo 2, Cirugía Palpebral Paso a Paso. Córdoba: Editorial MAC LINE, S. L., 2004;46-54.

6. Bodnar ZM, Neimkin M, Holds JB. Automated Ptosis Measurements From Facial Photographs, JAMA Ophthalmol. 2016;134:146-50.

7. Sacchidanand SA, Deepak HS, Vishal C, Revathy TN. Transcutaneous blepharoplasty in blepharochalasis, J Cutan Aesthet Surg. 2012;5:284-6.

8. Mendelson BC, Luo D. Secondary upper lid blepharoplasty: a clinical series using the tarsal fixation technique, Plast Reconstr Surg. 2015;135:508-16.
9. Novo A, Salvador JF, Lorda E, Laredo C. Corrección de ptosis palpebral por la incisión de blefaroplastía, Cir Plást Iberolatinoam. 2006;32:179-84.

10. Calderón W, Cerda C, Ford A, Israel G, Cabello R, Cornejo S, et al. Alternativa quirúrgica para la corrección del ectropión: Huincha tarsal. Rev Chil Cir. 2002;54:414-6.

11. Leatherbarrow B, Saha K. Complications of blepharoplasty, Facial Plast Surg. 2013;29:281-8

12. Mahroo OA, Hysi PG, Dey S, Gavin EA, Hammond CJ, Jones CA. Outcomes of ptosis surgery assessed using a patientreported outcome measure: an exploration of time effects, Br J Ophthalmol. 2014;98:387-90. 